\title{
Reflets
}

Revue d'intervention sociale et communautaire

\section{Enjeux de santé des aînés francophones vivant en situation minoritaire : une analyse différenciée selon les sexes}

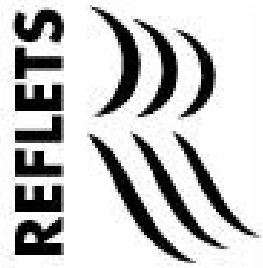

\section{Solange van Kemenade, Louise Bouchard et Christian Bergeron}

Volume 21, numéro 2, automne 2015

$20^{\mathrm{e}}$ anniversaire... déjà !

URI : https://id.erudit.org/iderudit/1035435ar

DOI : https://doi.org/10.7202/1035435ar

Aller au sommaire du numéro

Éditeur(s)

Reflets, Revue d'intervention sociale et communautaire

ISSN

1203-4576 (imprimé)

1712-8498 (numérique)

Découvrir la revue

Citer cet article

van Kemenade, S., Bouchard, L. \& Bergeron, C. (2015). Enjeux de santé des aînés francophones vivant en situation minoritaire : une analyse différenciée selon les sexes. Reflets, 21(2), 112-130. https://doi.org/10.7202/1035435ar
Résumé de l'article

La recherche en santé sur les Communautés de langue officielle vivant en situation minoritaire révèle des disparités. Le fait d'être minoritaire dans une société, conjointement avec d'autres déterminants, comme le statut socioéconomique, l'éducation en santé, et le fait de ne pas avoir un soutien social contribuent aux disparités de santé. Dans le contexte du vieillissement plus marqué des populations de langue officielle vivant en situation minoritaire, nous avons examiné la situation des aînés francophones. Pour cela, une analyse secondaire des données issues de l'Enquête sur la santé des collectivités canadiennes (ESCC) a été réalisée. Nous présentons dans cet article, une analyse différenciée de leur situation sociosanitaire selon le sexe et le genre en dressant le portrait des états de santé, des incapacités et des besoins sur le plan des soins de santé.
Tous droits réservés @ Reflets, Revue d’intervention sociale et communautaire, 2015
Ce document est protégé par la loi sur le droit d'auteur. L'utilisation des services d'Érudit (y compris la reproduction) est assujettie à sa politique d'utilisation que vous pouvez consulter en ligne. 


\section{Enjeux de santé des aînés francophones vivant en situation minoritaire : une analyse différenciée selon les sexes ${ }^{1}$}

Solange van Kemenade, $P h . D$.

Chercheuse associée à l'Université d'Ottawa, Institut de recherche sur la santé des populations, Professeure à temps partiel, Université du Québec en Outaouais et Université de Montréal

Louise Bouchard, Ph. D.

Professeure titulaire, École d'études sociologiques et anthropologiques et Institut de recherche sur la santé des populations, Université d'Ottawa et Institut de recherche de l'Hôpital Montfort

Christian Bergeron, Ph. D.

Professeur auxiliaire, École interdisciplinaire des sciences de la santé, Université d'Ottawa

\section{Résumé}

La recherche en santé sur les Communautés de langue officielle vivant en situation minoritaire révèle des disparités. Le fait d'être minoritaire dans une société, conjointement avec d'autres déterminants, comme le statut socioéconomique, l'éducation en santé, et le fait de ne pas avoir un soutien social contribuent aux disparités de santé. Dans le contexte du vieillissement plus marqué des populations de langue officielle vivant en situation minoritaire, nous avons examiné la situation des aînés francophones. Pour cela, une analyse secondaire des données issues de l'Enquête sur la santé des collectivités canadiennes (ESCC) a été réalisée. 
Nous présentons dans cet article, une analyse différenciée de leur situation sociosanitaire selon le sexe et le genre en dressant le portrait des états de santé, des incapacités et des besoins sur le plan des soins de santé.

Mots clés : aînés francophones, situation minoritaire, santé, accès aux soins, services de santé, sexe, langues officielles, Canada, Enquête de santé dans les collectivités canadiennes.

\section{Abstract}

Research into the health of official language minority communities unveils disparities. Being a minority in a society, together with other determinants such as socio-economic status, health education, and a lack of access to social support all contribute to disparities in health. Given that seniors from official language communities in a minority situation are living longer than ever, it is important to examine the situation of Francophone seniors. Based on data from the Canadian Community Health Survey (CCHS), we present in this article a differentiated sex- and genderbased analysis of their health and social situation with a focus on health conditions, disability and unmet needs.

Key words: Francophone seniors, minority situation, health, health care access, health services, sex, official languages, Canada, Canadian Community Health Survey

\section{Introduction}

Cet article porte un regard critique sur la santé des hommes et des femmes âgés de 65 ans ou plus et vivant en situation minoritaire au Canada. Soulignons l'importance d'un portrait différencié selon le sexe ou le genre, que la littérature documente bien en tant que déterminants des inégalités dans le domaine de la santé et de l'accès aux services. Les hommes et les femmes ne présentent pas les mêmes conditions de vie socioéconomiques, ni les mêmes 
"Si les femmes vivent plus longtemps que les hommes, les années supplémentaires sont parfois accompagnées de maladies chroniques et souvent vécues dans une plus grande pauvreté et dans un isolement social...» profils de santé physique ou mentale. Si les femmes vivent plus longtemps que les hommes, les années supplémentaires sont parfois accompagnées de maladies chroniques et souvent vécues dans une plus grande pauvreté et dans un isolement social (Bouchard, et collab., 2015).

L'analyse différenciée selon les sexes fournit des informations indispensables pour mieux orienter les politiques et la planification en matière de santé, et mieux adapter les soins aux besoins des individus (Gahagan, et collab., 2015). Cela contribue aussi à atteindre les objectifs d'équité en santé.

Le présent article propose d'abord un survol de la littérature canadienne récente sur la santé des aînés vivant en situation minoritaire et présente la méthodologie et les résultats de l'analyse secondaire de l'Enquête sur la santé des collectivités canadiennes (ESCC). Puis, nous y dressons un profil sociosanitaire des hommes et des femmes francophones âgés de 65 ans ou plus et vivant en situation minoritaire et nous examinons les écarts avec la population canadienne dans la même tranche d'âge comme population de référence. Nous proposons enfin quelques pistes d'interprétation ainsi que des suggestions pour la recherche future sur ce thème.

\section{L'état des connaissances sur la santé des aînés en situation linguistique minoritaire}

Au Canada, la population francophone vivant en situation linguistique minoritaire vieillit proportionnellement plus rapidement que l'ensemble de la population canadienne (Bouchard, et collab., 2015). Ce vieillissement s'est considérablement accentué au cours des dernières décennies et pour une grande proportion d'aînés francophones, surtout pour les femmes francophones, il s'accompagne d'une précarité financière. En effet, les aînés francophones sont plus susceptibles d'être sans diplôme d'études secondaires et de se situer dans le quintile de revenu le plus faible 
"...les aînés

francophones sont

plus susceptibles d'être

sans diplôme d'études

secondaires et de se

situer dans le quintile de revenu le plus faible comparativement aux aînés anglophones..." comparativement aux ainés anglophones ${ }^{2}$ (Bouchard, et collab., 2015). Dans cette même étude, les femmes francophones de 65 ans ou plus apparaissent plus vulnérables que les hommes du point de vue de leur niveau de revenu et d'éducation.

Bien que leur espérance de vie soit plus élevée que celle des hommes, les femmes passent aussi plus d'années en moins bonne santé (Luy, et Minagawa, 2014). Elles souffrent d'incapacités souvent associées à la douleur et au manque de mobilité et d'agilité (Statistique Canada, 2007). Cette situation entraîne des besoins particuliers et une demande accrue de services de santé qui ne sont pas toujours disponibles, comme signalé dans les recherches recensées ci-dessous.

Bernier et collab. (2009) se sont intéressés aux besoins ainsi qu'à l'offre des services destinés aux ainés francophones vivant en situation minoritaire. Ils ont mené une enquête qualitative dans dix villes du nord-est de l'Ontario. Les résultats de l'étude montrent un manque de services dans la langue de la minorité dans les régions rurales et éloignées. Ce qui aboutit à de moins bons résultats de santé et à des difficultés accrues pour accéder aux services de soins. Les auteurs concluent que ces lacunes alimentent chez les aînés un sentiment d'insécurité et de désillusion par rapport au système de santé de la province ontarienne.

Menée dans une communauté urbaine du NouveauBrunswick,l'étude de Dupuis-Blanchard et collab. (2013) en arrive à des résultats identiques. Les chercheuses ont analysé la situation des aînés francophones qui souhaitent vivre le plus longtemps possible dans leur domicile et ont conclu au manque de services et de soutien dans la communauté. Le vécu du vieillissement chez soi pour les personnes âgées francophones vivant en situation minoritaire exige, selon l'étude, des efforts concertés famillecommunauté-gouvernement.

Alimezelli et collab. (2013) présentent des conclusions similaires à celles de ces études qualitatives. Ils considèrent que les institutions des services de santé et les gouvernements répondent de façon inadéquate aux besoins de plus en plus nombreux d'accès aux services de santé pour les populations vieillissantes 
du Canada et notamment celles vivant en situation minoritaire. Selon les chercheurs, les barrières linguistiques auxquelles font face les aînés vivant en situation minoritaire affecteraient davantage l'autoévaluation de leur santé. En effet, les sentiments d'infériorité et de minorisation chez les aînés francophones pourraient affecter la perception de leur santé, étant donné la nature subjective de cet indicateur multidimensionnel (Bouchard, et Desmeules, 2013). Par ailleurs, la forte vitalité d'une communauté linguistiquement minoritaire a été associée à une meilleure santé autoévaluée par opposition à un degré de vitalité plus faible (Alimezelli, et collab., 2013).

Enfin, selon de Moissac et collab. (2012), seulement un quart de la population francophone au Manitoba accède à des services en français. Selon ces chercheuses et chercheurs, non seulement les francophones pourraient hésiter à s'identifier comme tels pour obtenir des services dans leur langue, mais aussi le manque de connaissance des ressources disponibles en français pourrait contribuer à cette perception de pénurie.

La difficulté à obtenir des services dans sa langue est un fait avéré variant selon le statut des langues officielles dans les différentes provinces canadiennes : $78 \%$ des répondantes ou répondants de Terre-Neuve-et-Labrador disent avoir un accès difficile aux services de santé dans la langue de la minorité. Les proportions sont de $72 \%$ en Saskatchewan, $71 \%$ en ColombieBritannique et $67 \%$ en Alberta. En Ontario et au Manitoba, la proportion de répondantes et de répondants est de $40 \%$, alors qu'au Québec elle est de $26 \%$. C'est au Nouveau-Brunswick, seule province officiellement bilingue, où la proportion de répondantes ou répondants disant avoir de la difficulté à obtenir des services dans leur langue est la plus faible (11\%) (GagnonArpin, et collab., 2014).

La disponibilité de services en français semble cruciale pour les personnes âgées vivant en situation minoritaire, car elles sont plus susceptibles de voir leur communication entravée par divers aspects de nature psychosociale. Parmi ces aspects, se retrouvent : le niveau spécialisé du langage médical;l'insécurité de ne pas être à la hauteur face aux difficultés de compréhension; le sentiment 
d'être sous stress du temps alloué à la rencontre médecin-patient; la difficulté de comprendre et de s'exprimer en anglais; et la difficulté d'exprimer l'émotion et la douleur dans une langue seconde (Bouchard, et collab., 2012).

Cependant, des difficultés pour accéder à des services dans la langue de la minorité, notamment pour les aînés qui souhaitent demeurer dans les collectivités rurales, sont signalées dans la littérature. Ces personnes peuvent être confrontées à des obstacles pour leur maintien à domicile et leur participation active au sein de la communauté (Turcotte, et Schellenberg, 2007; DupuisBlanchard, et collab., 2013; Forgues, et collab., 2011).

\section{La méthode}

Les analyses descriptives présentées dans cet article découlent des données de l'ESCC, l'enquête transversale qui recueille des informations sur l'état de santé,l'utilisation des soins de santé et les déterminants de la santé de la population canadienne.Étant donné la taille réduite de l'échantillon francophone dans les données de l'ESCC, plusieurs cycles d'enquêtes ont été combinés selon une méthodologie validée par Statistique Canada pour nous permettre de tirer des conclusions valides. Ainsi, cette combinaison s'avère nécessaire pour accroître les effectifs insuffisants des populations de petite taille (Makvandi, et collab., 2013). Cela dit, la faiblesse des échantillons rend impossible les analyses par régionales et selon le sexe pour la plupart des provinces canadiennes, à l'exception de l'Ontario, où la population francophone est plus importante.

Huit cycles de l'ESCC de 2003 à 2012 englobant 476593 répondants non francophones et 29221 francophones ont été utilisés pour l'analyse $e^{3}$. Pour définir la population francophone, un algorithme permettant de filtrer les individus a été créé à partir de quatre variables de langue présentes dans l'ESCC : la langue de conversation; la langue maternelle; la langue de l'entrevue; et la langue de contact préférée lors de l'enquête. Ainsi, tous les locuteurs francophones ont pu être identifiés. 
Des proportions pondérées ont été utilisées pour assurer que les données soient représentatives de la population ciblée.

\section{Les résultats}

"...la population âgée de 65 ans ou plus représente $15 \%$ de la population totale du Canada, alors que les aînés francophones représentent $18 \%$. Parmi les aînés francophones vivant en situation minoritaire, les femmes $(55 \%)$ sont plus nombreuses que les hommes (45\%)...»
Les résultats de notre analyse sont présentés de manière à établir le profil sociosanitaire des hommes et des femmes mis en perspective avec celui de la population canadienne totale âgée de 65 ans ou plus. Les caractéristiques sociodémographiques, les perceptions sur la santé et les incapacités, les comportements reliés à la santé, les besoins de services et les difficultés à les obtenir constituent les différentes rubriques.

\section{Les caractéristiques sociodémographiques des hommes et des femmes aînés francophones}

Sur le plan démographique, la population âgée de 65 ans ou plus représente $15 \%$ de la population totale du Canada, alors que les aînés francophones représentent $18 \%$. Parmi les aînés francophones vivant en situation minoritaire, les femmes (55\%) sont plus nombreuses que les hommes (45\%) (Tableau 1).

Les aînés francophones vivant en situation minoritaire détiennent dans leur ensemble un niveau de scolarité plus bas que l'ensemble des aînés au Canada et sont plus nombreux à résider dans les zones rurales. En ce qui concerne les différences entre les hommes et les femmes, ces dernières sont proportionnellement plus nombreuses que les hommes à ne pas avoir un diplôme d'études secondaires (37\% et $31 \%$ respectivement), à avoir un faible revenu (36\% et $24 \%$ respectivement) et à vivre seules (38\% et $19 \%$ respectivement) (Tableau 1). Par contre, les hommes francophones sont plus nombreux que les femmes à habiter en milieu rural (32\% et $27 \%$ respectivement).

Les francophones d'origine immigrante sont sous-représentés comparativement à l'ensemble de la population âgée de 65 ans ou plus (32\% versus $16 \%$, dont $7 \%$ des femmes francophones et $9 \%$ des hommes francophones). 
Tableau 1 - Caractéristiques sociodémographiques

\begin{tabular}{|l|c|c|c|}
\hline & \multirow{2}{*}{$\begin{array}{c}\text { 65 ans+ Canada } \\
\text { (\%) }\end{array}$} & \multicolumn{2}{|c|}{ Population francophone } \\
\cline { 3 - 4 } & & $\begin{array}{c}\text { Hommes } \\
\text { francophones (\%) }\end{array}$ & $\begin{array}{c}\text { Femmes } \\
\text { francophones (\%) }\end{array}$ \\
\hline Hommes & 45 & & \\
\hline Femmes & 55 & & 20 \\
\hline 65 ans + & 15 & 16 & 59 \\
\hline $65-74$ ans & 57 & 63 & 33 \\
\hline $75-84$ ans & 34 & 31 & 9 \\
\hline 85 ans et plus & 9 & 6 & 37 \\
\hline Sans diplôme d'études secondaires & 23 & 31 & 36 \\
\hline Faible revenu (1'r quintile) & 28 & 24 & 38 \\
\hline Vit seul & 28 & 19 & 27 \\
\hline Milieu rural & 20 & 32 & 7 \\
\hline Statut d'immigrant & 32 & 9 & \\
\hline
\end{tabular}

Source des données : ESCC : 2.1 (2003), 3.1 (2005), 4.1 (2007), 2008, 2009, 2010, 2011, 2012. Canada excluant le Québec et les Territoires. Données pondérées tirées d'un échantillon de 7640 Canadiens francophones et de 157312 Canadiens âgés de 65 ans ou plus.

"Les hommes francophones âgés de 65 ans ou plus (28\%) sont plus susceptibles de se déclarer en mauvaise santé physique que les femmes francophones du même groupe d'âge $(25 \%)$ et que la moyenne canadienne (25\%). De même, $7 \%$ des femmes et $6 \%$ des hommes se déclarent en mauvaise santé mentale. »

\section{L'état de santé chez les hommes et les femmes aînés francophones}

La perception de la santé et d'autres indicateurs, tels que le besoin d'aide pour effectuer ses tâches quotidiennes et la déclaration des limitations des activités dues à une condition chronique, constituent des indicateurs utiles permettant d'évaluer assez justement l'état de santé ainsi que les besoins de services et de soins d'une population.

Les hommes francophones âgés de 65 ans ou plus (28\%) sont plus susceptibles de se déclarer en mauvaise santé physique que les femmes francophones du même groupe d'âge (25\%) et que la moyenne canadienne (25\%). De même, $7 \%$ des femmes et $6 \%$ des hommes se déclarent en mauvaise santé mentale.

Par ailleurs, les femmes aînées sont plus susceptibles que les hommes d'avoir besoin d'aide pour accomplir les tâches 
quotidiennes (33\% et $21 \%$ respectivement) et par rapport à la moyenne canadienne (27\%) (Tableau 2).

\section{Le style de vie}

Le surpoids et la sédentarité constituent deux facteurs déterminants de maladies chroniques et ils affectent plus de la moitié des aînés francophones vivant en situation minoritaire. Les femmes francophones sont proportionnellement moins nombreuses à être en surpoids que les hommes (56\% et $61 \%$ respectivement) et par rapport à la moyenne canadienne (57\%). Cependant, elles sont plus nombreuses que les hommes francophones à être inactives physiquement (62\% et $48 \%$ respectivement) et par rapport à la moyenne canadienne (57\%) (Tableau 2).

Tableau 2 - Santé perçue, limitations des activités et styles de vie

\begin{tabular}{|c|c|c|c|}
\hline & \multirow{2}{*}{$\begin{array}{c}65 \text { ans+ Canada } \\
\text { (\%) }\end{array}$} & \multicolumn{2}{|c|}{ Population francophone } \\
\hline & & $\begin{array}{c}\text { Hommes } \\
\text { francophones (\%) }\end{array}$ & $\begin{array}{c}\text { Femmes } \\
\text { francophones (\%) }\end{array}$ \\
\hline Mauvaise santé perçue & 25 & 28 & 25 \\
\hline Mauvaise santé mentale perçue & 6 & 7 & 6 \\
\hline Besoin d'aide pour effectuer ses tâches & 27 & 21 & 33 \\
\hline Limitations des activités à la maison & 37 & 32 & 36 \\
\hline Surpoids-obèse & 57 & 61 & 56 \\
\hline Inactif physiquement & 57 & 48 & 62 \\
\hline Consomme moins de 5 fruits \& légumes & 55 & 62 & 49 \\
\hline Fumeurs réguliers \& occasionnels & 10 & 12 & 9 \\
\hline Buveurs quotidiens & 22 & 26 & 12 \\
\hline
\end{tabular}

Source des données : ESCC : 2.1 (2003), 3.1 (2005), 4.1 (2007; 2008; 2009; 2010; 2011; 2012). Canada excluant le Québec et les Territoires. Données pondérées tirées d'un échantillon de 7640 Canadiens francophones et de 157312 Canadiens âgés de 65 ans ou plus.

Quant aux habitudes de vie saine qui contribuent à améliorer ou à maintenir la santé, les hommes francophones ont de moins bonnes habitudes de vie que les femmes francophones. En effet, ils sont moins susceptibles de consommer la portion quotidienne 
"Dans le cadre

des maladies

chroniques examinées,

l'hypertension

artérielle, l'asthme, les maux de dos, les maladies cardiaques et le diabète sont les problèmes de santé les plus importants à affecter les aînés francophones vivant en situation minoritaire..." recommandée de fruits et légumes ${ }^{4}$, sont presque une fois et demie plus nombreux que les femmes francophones à fumer régulièrement ou occasionnellement des cigarettes et deux fois plus nombreux à boire régulièrement de l'alcool.

\section{Les maladies chroniques}

Les problèmes de santé de longue durée (ou maladies chroniques) sont plus fréquents chez les aînés francophones ciblés dans notre analyse. Ils sont définis dans l'ESCC comme « un état qui dure ou devrait durer six mois ou plus et qui a été diagnostiqué par un professionnel de la santé $»^{5}$. Dans le cadre des maladies chroniques examinées, l'hypertension artérielle, l'asthme, les maux de dos, les maladies cardiaques et le diabète sont les problèmes de santé les plus importants à affecter les aînés francophones vivant en situation minoritaire (Tableau 3).

Tableau 3 - Problèmes de santé chroniques

\begin{tabular}{|l|c|c|c|}
\hline & \multirow{2}{*}{$\begin{array}{c}\mathbf{6 5} \text { ans+ Canada } \\
\text { (\%) }\end{array}$} & \multicolumn{2}{|c|}{ Population francophone } \\
\cline { 3 - 4 } & & $\begin{array}{c}\text { Hommes } \\
\text { francophones (\%) }\end{array}$ & $\begin{array}{c}\text { Femmes } \\
\text { francophones (\%) }\end{array}$ \\
\hline Asthme & 45 & 38 & 53 \\
\hline Maux de dos (*) & 28 & 26 & 28 \\
\hline Hypertension artérielle & 48 & 42 & 50 \\
\hline Diabète & 17 & 21 & 15 \\
\hline Maladie cardiaque & 18 & 22 & 17 \\
\hline Cancer & 7 & 9 & 6 \\
\hline Ulcère d'estomac & 4 & 5 & 6 \\
\hline Accident vasculaire cérébral (AVC) & 5 & 5 & 4 \\
\hline Troubles de l'humeur & 6 & 4 & 7 \\
\hline Anxiété & 4 & 3 & 7 \\
\hline
\end{tabular}

( ) Autres que ceux dus à la fibromyalgie et l'arthrite.

Source des données : ESCC : 2.1 (2003), 3.1 (2005), 4.1 (2007; 2008; 2009; 2010; 2011; 2012). Canada excluant le Québec et les Territoires. Données pondérées tirées d'un échantillon de 7640 Canadiens francophones et de 157312 Canadiens âgés de 65 ans ou plus. 
Certaines différences ressortent lorsque nous comparons la situation des hommes francophones et celle des femmes francophones. En effet, les femmes âgées sont presque une fois et demie plus nombreuses à souffrir d'asthme $(53 \%$ et $38 \%$ respectivement), légèrement plus nombreuses à avoir de maux de dos $(28 \%$ et $26 \%$ respectivement) et de l'hypertension artérielle (50\% et $42 \%$ respectivement) (Tableau 3). Par contre, le diabète affecte davantage les hommes que les femmes francophones $(21 \%$ et $15 \%$ respectivement), ainsi que les maladies cardiaques (22\% et $17 \%$ respectivement), le cancer ( $9 \%$ et $6 \%$ respectivement) et l'accident vasculaire cérébral (AVC) (5\% et $4 \%$ respectivement). Par contre, les femmes sont près de deux fois plus nombreuses que les hommes à être affectées par des troubles de l'humeur $(7 \%$ et $4 \%$ respectivement) et de l'anxiété (7\% et $3 \%$ respectivement) ${ }^{6}$.

\section{Les besoins, l'utilisation des soins de santé et la qualité des services de soins de santé}

La très grande majorité des aînés francophones vivant en situation minoritaire ont besoin de soins de routine (69\%); plus d'un quart ont eu besoin d'une hospitalisation au cours de 12 mois précédant l'enquête, et $34 \%$ ont eu besoin des services spécialisés. Néanmoins, $95 \%$ de ces aînés déclarent avoir un médecin régulier et être dans la grande majorité satisfaits des soins reçus (93\%). Il faut signaler que les aînés francophones vivant en situation minoritaire sont légèrement moins nombreux que l'ensemble des aînés canadiens à avoir un médecin de famille (95\%) et que $38 \%$ de ces aînés parlent en français avec leur médecin (Tableau 4).

Les différences entre les hommes et les femmes francophones ne sont pas très marquées, mais ces dernières déclarent avoir, en général, moins besoin de services et d'informations relatives à la santé, mais elles ont plus de difficultés à obtenir des soins. Par contre, les hommes sont plus nombreux que les femmes à déclarer avoir besoin de services spécialisés (39\% et $31 \%$ respectivement), d'une chirurgie non urgente (12\% et $11 \%$ respectivement), de l'information sur la santé (30\% et $29 \%$ respectivement), de soins de routine ( $73 \%$ et $66 \%$ respectivement) et de soins immédiats 
(20\% et $19 \%$ respectivement). Les femmes, par contre, sont plus nombreuses à manifester des difficultés à obtenir des services spécialisés (18\% et $14 \%$ respectivement), une chirurgie non urgente (14\% et $11 \%$ respectivement), des soins immédiats (15\% et $11 \%$ respectivement), et deux fois plus nombreuses à recevoir des soins à domicile (20\% et $10 \%$ respectivement). Les hommes et les femmes francophones ont déclaré dans une proportion égale (34 \%) avoir eu besoin d'une hospitalisation au cours de l'année précédant l'enquête ${ }^{7}$.

Tableau 4 - Besoins, accès et qualité des services de soins de santé

\begin{tabular}{|l|c|c|c|}
\hline & \multirow{2}{*}{$\begin{array}{c}\text { ans+ Canada } \\
\text { (\%) }\end{array}$} & \multicolumn{2}{|c|}{ Population francophone } \\
\cline { 3 - 4 } & & $\begin{array}{c}\text { Hommes } \\
\text { francophones (\%) }\end{array}$ & $\begin{array}{c}\text { Femmes } \\
\text { francophones (\%) }\end{array}$ \\
\hline A un médecin régulier & 96 & 94 & 95 \\
\hline $\begin{array}{l}\text { Parle habituellement français avec le } \\
\text { médecin }\end{array}$ & 2 & 38 & 38 \\
\hline Besoin de services spécialisés & 39 & 39 & 31 \\
\hline Besoin de chirurgie non urgente & 11 & 12 & 11 \\
\hline Besoin d'informations sur la santé & 39 & 30 & 29 \\
\hline Besoin de soins de routine & 64 & 73 & 66 \\
\hline Besoins de soins immédiats & 21 & 20 & 19 \\
\hline $\begin{array}{l}\text { Difficulté à obtenir des services } \\
\text { spécialisés }\end{array}$ & 18 & 14 & 18 \\
\hline $\begin{array}{l}\text { Difficulté à obtenir une chirurgie non } \\
\text { urgente }\end{array}$ & 16 & 11 & 14 \\
\hline Difficulté à obtenir de l'information & 12 & 12 & 10 \\
\hline Difficulté à obtenir des soins de routine & 9 & 7 & 7 \\
\hline Difficulté à obtenir des soins immédiats & 16 & 12 & 15 \\
\hline Soins à domicile & 14 & 10 & 20 \\
\hline Hospitalisation & 35 & 34 & 34 \\
\hline Satisfaits des soins reçus & 94 & 94 & 92 \\
\hline
\end{tabular}

Source des données : ESCC : 2.1 (2003), 3.1 (2005), 4.1 (2007; 2008; 2009; 2010; 2011; 2012). Canada excluant le Québec et les Territoires. Données pondérées tirées d'un échantillon de 7640 Canadiens francophones et de 157312 Canadiens âgés de 65 ans ou plus. 
Enfin, les femmes francophones aînées vivant en situation minoritaire sont moins satisfaites des soins reçus comparativement aux hommes francophones aînés (92\% et $94 \%$ respectivement), mais elles demeurent quand même très majoritairement satisfaites des soins qu'elles ont reçus (92\%).

Le besoin de soins de routine est plus élevé chez les aînés francophones comparativement à l'ensemble des aînés au Canada, alors que ces derniers ont des besoins plus élevés à obtenir une chirurgie non urgente ainsi que des informations reliées à la santé.

\section{La discussion}

«...les femmes

francophones semblent plus vulnérables d'un point de vue socioéconomique, elles ont proportionnellement des niveaux d'études et de revenus plus faibles que les hommes francophones et sont plus nombreuses à vivre seules. "
Certains constats se dégagent de notre analyse des données de l'ESCC. Le premier concerne certains écarts entre les hommes et les femmes francophones vivant en situation minoritaire. Dans ce sens, les femmes francophones semblent plus vulnérables d'un point de vue socio-économique, elles ont proportionnellement des niveaux d'études et de revenus plus faibles que les hommes francophones et sont plus nombreuses à vivre seules. Ces résultats sont par ailleurs convergents avec certaines des études examinées dans le cadre de notre revue de littérature (Bouchard, et collab., 2015).

De plus, et comme signalé dans les résultats, les aînées francophones déclarent en plus grande proportion des limitations pour effectuer les activités quotidiennes, comparativement aux hommes francophones. Elles sont aussi plus nombreuses que les hommes à avoir besoin d'aide pour effectuer ces tâches et à recevoir des soins à domicile. Toutefois, $30 \%$ des hommes francophones habitent en milieu rural, ce qui rend plus difficiles leurs déplacements et leur accès à des soins de santé.

Les hommes francophones sont légèrement plus nombreux que les femmes à déclarer une mauvaise santé physique et mentale. Cette perception de leur santé s'accompagne de conditions objectives, telles que des comportements moins sains comparativement aux femmes et la présence proportionnellement plus élevée de maladies chroniques, comme le diabète qui 
est étroitement lié aux habitudes de vie. En effet, ils sont moins susceptibles que les femmes de consommer la portion quotidienne recommandée de fruits et de légumes, et plus susceptibles qu'elles de fumer et de consommer régulièrement des boissons alcoolisées.

Lorsque nous comparons les aînés francophones vivant en situation minoritaire avec l'ensemble de la population âgée de 65 ans ou plus au Canada, les premiers sont légèrement plus nombreux à déclarer une mauvaise santé physique. Les hommes francophones sont aussi légèrement plus nombreux que les nonfrancophones à déclarer une mauvaise santé mentale autoperçue. Les raisons qui pourraient expliquer ce phénomène ont été évoquées dans une section précédente et incluent les barrières linguistiques qui affecteraient l'autoévaluation de la santé de la part des aînés (Alimezelli, et collab., 2013) ainsi qu'une intériorisation du fait minoritaire qui pourrait affecter la perception de la santé (Bouchard, et Demeules, 2013).

Lorsque nous examinons les besoins de santé, l'accès aux soins de santé et la satisfaction avec les soins reçus, il ne semble pas y avoir de grands écarts entre les ainés francophones vivant en situation minoritaire et l'ensemble des aînés canadiens. Par contre, les premiers sont plus nombreux à déclarer un besoin de soins de routine et des soins à domicile (Tableau 4). En ce qui concerne ces derniers, les femmes sont plus nombreuses que les hommes à les recevoir, et les femmes francophones davantage. Selon la définition de Statistique Canada, les services à domicile incluent les soins de santé, des services d'entretien ménager ou d'autres services de soutien reçus à domicile. Les gens pourraient recevoir de tels services en raison d'un problème de santé ou d'un état de santé influant sur leurs activités quotidiennes.

Répondre aux besoins de ces femmes âgées, en soins à domicile, constitue une priorité, car les études démontrent que les personnes ayant des besoins non comblés en matière d'aide ou de soins à domicile sont plus susceptibles d'avoir des états émotionnels négatifs, de ressentir un stress élevé, d'avoir des problèmes de sommeil et d'éprouver un sentiment de solitude (Turcotte, 2014).

Enfin, les aînés francophones vivant en situation minoritaire sont très majoritairement satisfaits des soins reçus, mais légèrement 
moins nombreux que l'ensemble des aînés canadiens. Les femmes francophones vivant en situation minoritaire constituent le groupe le moins satisfait des soins reçus.

\section{Conclusion}

"Les données récentes issues de l'ESCC et que nous avons analysées dans le cadre du présent article confirment le maintien d'un léger écart dans certains indicateurs entre les aînés francophones vivant en situation minoritaire et l'ensemble de la population âgée de 65 ans ou plus au Canada."
Dans le contexte actuel du vieillissement de la population et d'une manière particulière, de la population francophone vivant en situation minoritaire au Canada, il est important d'analyser l'état de santé des personnes âgées et d'identifier leurs besoins en matière de soins de santé.

Les données récentes issues de l'ESCC et que nous avons analysées dans le cadre du présent article confirment le maintien d'un léger écart dans certains indicateurs entre les aînés francophones vivant en situation minoritaire et l'ensemble de la population âgée de 65 ans ou plus au Canada.

Cette analyse nous a permis d'identifier les sphères de vulnérabilité en santé spécifiques à chaque sexe et nous permet de conclure que les femmes aussi bien que les hommes francophones vivant en situation minoritaire devraient être considérés dans l'élaboration des politiques, des programmes de santé et dans l'offre de services dans leur langue. D'une part, plus touchées sur le plan socio-économique, les femmes déclarent se porter mieux que les hommes quant à la perception de leur santé, mais elles sont plus limitées dans leurs activités et affectées par les problèmes qui touchent à la santé mentale (troubles de l'humeur et de l'anxiété). D'autre part, les hommes vivant en milieu rural et souffrant de maladies comme le diabète se déclarent en moins bonne santé que les autres hommes. Les différences selon le sexe mériteraient d'être davantage examinées dans des analyses plus approfondies. De plus, des recherches futures pourraient explorer, entre autres, les facteurs protecteurs et de risque dans le cas de maladies chroniques ainsi que ces facteurs chez les personnes vivant en milieu rural ou isolées. 
"Identifier les groupes les plus vulnérables au sein des populations minoritaires est aussi un besoin incontournable dans un contexte où les décisions doivent s'appuyer sur des données probantes et qui se caractérise par la rareté des ressources. "
Mettre en lumière les différences entre les populations selon leur statut minoritaire ou majoritaire est important pour mieux en connaitre l'impact sur la santé des aînés francophones. Les portraits de santé de la population francophone vivant en situation minoritaire, comme celui que nous présentons dans le présent article, aident à combler le manque de données qui s'y rapportent.

Ces connaissances deviennent cruciales pour informer sur le sujet et pour influencer la planification et la politique des soins de santé en français. Identifier les groupes les plus vulnérables au sein des populations minoritaires est aussi un besoin incontournable dans un contexte où les décisions doivent s'appuyer sur des données probantes et qui se caractérise par la rareté des ressources.

\section{Des pistes de recherche}

Il existe un besoin de mieux connaitre comment les hommes et les femmes francophones vivant en situation minoritaire se démarquent sur le plan de leurs états de santé en général, et des comportements adoptés pour rester en santé. De plus, certaines questions doivent être mieux examinées afin de générer des connaissances plus approfondies qui puissent aider à mieux planifier les services de santé et services sociaux. Par exemple, les soins et le maintien à domicile, l'offre active de services dans la langue de la minorité et les services offerts aux ainés francophones devraient être considérés dans les priorités de recherche ainsi que dans les programmes politiques.

Par rapport au maintien à domicile, nous savons que les femmes francophones ont plus tendance à déclarer des limitations pour réaliser les activités quotidiennes, comparativement aux hommes francophones. Elles sont aussi plus nombreuses que les hommes à avoir besoin d'aide pour effectuer ces tâches et à recevoir des soins à domicile, mais nous ne connaissons pas, par exemple, quels types de besoins ni quels types de soins leur sont spécifiquement adressés (soins infirmiers, aide pour prendre un bain, aide pour l'entretien ménager, services de relève, préparation de repas, etc.). Lorsqu'il s'agit des besoins non comblés, il serait nécessaire de connaître les raisons pour lesquelles les services n'ont pas été offerts, ainsi que 
"...dans une perspective de genre, d'autres études pourraient contribuer à mieux comprendre les habitudes de vie des personnes âgées, leurs connaissances par rapport aux choix d'habitudes de vie saine et le sens qu'elles attribuent à la santé, à la maladie et aux moyens qu'elles privilégient pour rester en santé." le recours aux services des agences privées. Dans ce sens, l'ESCC fournit beaucoup d'information qui pourrait être utilisée quant aux services à domicile.

En outre, les études ethnographiques réalisées auprès des aînés francophones ont permis d'importantes contributions relatives à certains thèmes, tels celui de l'offre de services aux aînés dans la langue de la minorité (Bernier, et collab., 2009; de Moissac, et collab., 2012), celui du maintien à domicile et des services adressés aux aînés francophones (Dupuis-Blanchard, et collab., 2013) et celui des enjeux de la littératie en santé (Bouchard, et collab., 2012). Dans cette même tradition de recherche et dans une perspective de genre, d'autres études pourraient contribuer à mieux comprendre les habitudes de vie des personnes âgées, leurs connaissances par rapport aux choix d'habitudes de vie saine et le sens qu'elles attribuent à la santé, à la maladie et aux moyens qu'elles privilégient pour rester en santé.

Les études qualitatives pourraient également mettre en valeur les différences locales et régionales, masquées lorsque les populations sont de petite taille et leur nombre insuffisant pour produire des analyses quantitatives. Ce type d'étude permettrait enfin de comprendre les réalités contextuelles et améliorer à l'échelle locale les services dans la langue de la minorité (Alimezelli, et collab., 2013).

En prenant compte des dimensions liées au genre, les analyses fondées sur les enquêtes de santé nationales et provinciales contribueraient à réduire les écarts sur le plan des inégalités non seulement entre les minorités de langues officielles et la société majoritaire, mais aussi entre les hommes et les femmes.

Finalement, au cours des prochaines années, il serait important de mieux comprendre le vieillissement de la population francophone vivant en situation minoritaire et d'approfondir les différences de genre. Ces thèmes ont été très peu explorés jusqu'ici et étant donné que la population âgée francophone est diversifiée et a des besoins de santé non comblés, il est important d'adapter nos politiques et pratiques en conséquence. 


\section{Notes}

1 Cette étude a été rendue possible grâce au soutien financier du ministère de la Santé et des Soins de longue durée de l'Ontario et de son Initiative de réseaux de recherche appliquée en santé, le Réseau de recherche appliquée sur la santé des francophones de l'Ontario (RRASFO). Nous tenons à remercier Ewa Sucha, responsable du traitement des données de l'ESCC. Les analyses ont été réalisées au Centre de données de Carleton, Ottawa, Outaouais (CDR-COO), membre du Réseau canadien des Centres de données de recherche (RCCDR). Les idées exprimées dans ce texte n'engagent que ses auteurs.

2 Selon l'Enquête sur les dépenses des ménages de 2012 de Statistique Canada, les foyers se situant sur le premier quintile disposaient d'un revenu annuel moyen de $17300 \$$ (Conseil de la radiodiffusion et des télécommunications canadiennes, 2013). Conseil de la radiodiffusion et des télécommunications canadiennes. Quelles sont les caractéristiques d'un foyer canadien moyen? http://www.crtc.gc.ca/fra/publications/reports/policymonitoring/2013/cmr2.htm Consulté le 14 février 2015.

3 Cycles 2.1 (2003), 3.1 (2005), 4.1 (2007; 2008; 2009; 2010; 2011; 2012).

4 Selon le Guide alimentaire canadien, il est recommandable pour les adultes de 51 ans et plus sept portions des fruits et des légumes par jour en moyenne. Se référer à : http://www.hc-sc. gc.ca/fn-an/food-guide-aliment/choose-choix/fruit/serving-portion-fra.php Consulté le 14 février 2015.

5 ESCC, questionnaire 2012.

6 Les troubles de l'humeur incluent entre autres la dépression, le trouble bipolaire, la manie ou la dysthymie. Les troubles d'anxiété incluent entre autres les phobies, troubles obsessionnelscompulsifs ou un trouble de panique.

${ }^{7}$ Cette hospitalisation incluait : urgence, chirurgie d'un jour, clinique externe ou hospitalisation d'une nuit.

\section{Bibliographie}

ALIMEZELLI, Hubert T., et collab. (2013). "Determinants of self-rated health of Francophone seniors in a minority situation in Canada ", Minorités linguistiques et société / Linguistic Minorities and Society, Vol. 3, p. 144-170.

BERNIER, Christiane, et collab. (2009). « Citoyens de deuxième classe? Perceptions de la santé et du système de soins chez les francophones du nord-est de l'Ontario ", Francophonies d'Amérique, Vol. 28, p. 115-138.

BOUCHARD, Louise, et collab. (2015). «Précarité des populations francophones âgées vivant en situation linguistique minoritaire » Minorités linguistiques et société /Linguistic Minorities and Society, Vol. 6, p. 66-81.

BOUCHARD, Louise, et Martin DESMEULES (2013). "Les minorités linguistiques du Canada et la santé ", Healthcare Policy/Politiques de santé, numéro spécial,Vol. 9, p. 38-47.

BOUCHARD, Louise, et collab. (2013). «Language as an important determinant of poverty in the aging Francophone minority population in Canada ", The International Journal of Aging and Society, Vol. 2, p. 61-76. 
BOUCHARD, Louise, et collab. (2012). «Les Franco-Ontariens âgés souffrant de maladies chroniques se perçoivent-ils bien desservis? Une étude exploratoire de l'impact de la situation linguistique minoritaire ", Canadian Family Physician/Le médecin de famille canadien,Vol. 58, № 12, p. 1365, réf. du 14 février 2015, http://www.cfp.ca/content/58/12/1325.full

CONSEIL DE LA RADIODIFFUSION ET DESTÉLÉCOMMUNICATIONS CANADIENNES (2013). Quelles sont les caractéristiques d'un foyer canadien moyen?, réf. du 14 février 2015, http://www.crtc.gc.ca/fra/publications/reports/policymonitoring/2013/cmr2.htm

DE MOISSAC, Danielle, et collab. (2012). «Défis associés à l'offre de services de santé et de services sociaux en français au Manitoba : perceptions des professionnels ", Reflets : revue d'intervention sociale et communautaire, Vol. 18, No 2, p. 66-100.

DUPUIS-BLANCHARD, Suzanne, et collab. (2013). " La perception des aînés francophones en situation minoritaire face aux défis et aux enjeux liés au maintien à domicile en milieu urbain néo-brunswickois ", Canadian Journal of Public Health, Vol. 104 (6 SUPPL.1), p. S71-S74.

FORGUES, Éric, Michel DOUCET et Josée GUIGNARD NOËL (2011). "L'accès des aînés francophones aux foyers de soins en milieu minoritaire, un enjeu linguistique en santé et mieuxêtre ", Canadian Journal on Aging, Vol. 30, № 4, p. 603-616.

GAHAGAN,Jaqueline, Kimberley GRAY et Ardath WHYNACHT (2015). «Sex and gender matter in health research: addressing health inequities in health research reporting ", International Journal For Equity In Health, Vol. 14, № 1, p. 12-12.

GAGNON-ARPIN, Isabelle, et collab. (2014). "Accès et utilisation des services en langue minoritaire ", dans Rodrigue Landry (dir.), La vie dans une langue officielle minoritaire au Canada, Québec, Presses de l'Université Laval, p.193-219.

LUY, Marc, et Yuka MINAGAWA (2014). " Gender gaps - Life expectancy and proportion of life in poor health ", Health Reports, Vol. 25, №. 12, p. 12-19.

MAKVANDI, Ewa, et collab. (2013). " Methodological issues in analyzing small populations using CCHS cycles based on the official language minority studies ", Canadian Journal of Public Health, Vol. 104, N 6, p. S55-S59.

SANTÉ CANADA (2011). Guide alimentaire canadien, réf. Du 14 février 2015, http://www.hc-sc.gc.ca/fn-an/food-guide-aliment/choose-choix/fruit/serving-portion-fra. php.

STATISTIQUE CANADA (2007). L'Enquête sur la participation et les limitations d'activités de 2006 : tableaux. Ottawa, Statistique Canada ( $\mathrm{n}^{\circ}$. de cat. 89-628-XIF - $\left.\mathrm{n}^{\circ} 003\right)$.

TURCOTTE, Martin, et Grant SCHELLENBERG (2007). Un portrait des aînés au Canada, Ottawa, ON, Ministre de l'Industrie, réf. du 14 février 2015, http://www5.statcan.gc.ca/bsolc/olc-cel/olc-cel?catno=89-519-XIF\&lang=fra.

TURCOTTE, Martin (2014). Les Canadiens dont les besoins en soins à domicile sont non comblés, Statistique Canada, catalogue no. 75-006-X ISSN 2291-0859. 